\title{
Hospitalization due to respiratory syncytial virus infection in patients under 2 years of age with hemodynamically significant congenital heart disease
}

\author{
Silvia Andres, ${ }^{1}$ Gabriela Bauer, ${ }^{1}$ Susana Rodríguez, ${ }^{2}$ \\ Luis Novali, ${ }^{1}$ Diego Micheli, ${ }^{3}$ Diana Fariña ${ }^{4}$
}

\begin{abstract}
Objective: To describe hospitalization rates, burden of disease, and associated risk factors of acute respiratory infections (ARI), particularly those caused by respiratory syncytial virus (RSV) and non-RSV-ARI, in a cohort of patients under 2 years of age with congenital heart disease (CHD).

Methods: A prospective, observational cohort study was conducted with CHD patients discharged from the neonatal unit and followed up at a referral center. Demographic variables, type of $\mathrm{CHD}$, and medical needs were recorded. Study primary outcome was hospitalization for ARI (overall, due to RSV, and due to other causes). Secondary outcome was burden of disease in hospitalized patients. Incidence rates of hospitalization were calculated for overall ARI and RSV-ARI. Incidence densities were additionally calculated.

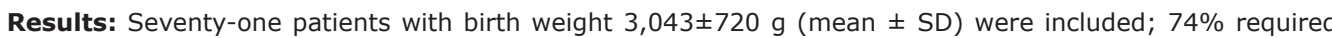
surgery and $8.4 \%$ died of CHD during the study. Overall, $22 / 71$ patients were hospitalized due to ARI $(31 \% ; 95 \% \mathrm{CI}$ $20-43), 15$ of them RSV-associated (21\%; 95\% CI 12-32), and there were 1.35 episodes of hospitalization for ARI/1,000 days of follow-up ( 0.92 episodes of hospitalization for RSV-ARI/1,000 days). Forty per cent of patients with ARI due to RSV needed admission to pediatric ICU and 30\% required mechanical ventilation vs. none in non-RSV-ARI.

Conclusions: In the studied population, ARI hospitalization was common, and RSV was its most frequent cause. Disease burden associated with RSV-ARI was considerable, although no patient died from ARI. Except younger age, no other biological or social risk factors were found associated with RSV-ARI hospitalization.
\end{abstract}

J Pediatr (Rio J). 2012;88(3):246-52: Respiratory syncytial virus, respiratory tract infections, heart disease, infants.

\section{Introduction}

Respiratory syncytial virus (RSV) is the most frequent cause of lower acute respiratory infection (ARI) in young children, and its most common clinical presentation is bronchiolitis. ${ }^{1-3}$

The Ministry of Health of Argentina said that ARI is the third leading cause of infant mortality, after perinatal and congenital malformations. Moreover, it is the first cause of consultation for children under 2 years old and represents over $40 \%$ of hospital discharges in the winter months. ${ }^{4}$

There is mounting evidence that bronchiolitis caused by RSV is associated with more severe respiratory events. ${ }^{5}$ There are pediatric populations at increased risk of morbidity and mortality from RSV infection ${ }^{6}$; children born with congenital heart disease (CHD) comprise one

1. Assistant pediatrician of the Neonatal Follow Up Program, Hospital Garrahan, Buenos Aires, Argentina

2. Research department coordinator, Hospital Garrahan, Buenos Aires, Argentina.

3. Cardiologist of the Neonatal Follow Up Program, Hospital Garrahan, Buenos Aires, Argentina.

4. Chief, Neonatal Intensive Care Unit, Hospital Garrahan, Buenos Aires, Argentina.

Conflicts of interest: Dr. Silvia Andres received financial support from Abbott Laboratories Inc. during study completion. Abbott Laboratories Inc. did not participate in study design and conclusions or in data collection and analysis nor any other form whatsoever. Neither Dr. Andres nor other author receives ongoing financial support nor has commercial interest with Abbott Laboratories Inc.

Suggested citation: Andres S, Bauer G, Rodríguez S, Novali L, Micheli D, Fariña D. Hospitalization due to respiratory syncytial virus infection in patients under 2 years of age with hemodynamically significant congenital heart disease. J Pediatr (Rio J). 2012;88(3):246-52.

Manuscript submitted Dec 15 2011, accepted for publication Mar 282012.

http://dx.doi.org/10.2223/JPED.2202 
of these populations. Several reports indicate increased susceptibility to ARI in children with CHD, especially in those with significant hemodynamic compromise, with higher rate of admission to intensive care units and higher risk of mortality than the general pediatric population. Among ARI etiologic agents in this group, RSV causes higher morbidity than other respiratory viruses. ${ }^{7-9}$

Approximately 5,000 children with CHD are born each year in Argentina. Seventy percent (70\%) of them will require surgical intervention before the first year of life. ${ }^{10}$ While waiting for either complete correction or palliative surgery, these patients persist with hemodynamic compromise, with or without pulmonary hypertension, and generally require cardiovascular drug therapy. ${ }^{11}$ Survival of children with $\mathrm{CHD}$ has dramatically increased; however, there is scarce or lacking data on the incidence and impact of ARI - both global and RSV-associated -, in particular during the first years, when CHD results in persistent hemodynamic compromise.

The use of the monoclonal antibody palivizumab for prevention of severe ARI caused by RSV in children less than 2 years of age with CHD is now the standard practice of care in many countries all over the world. ${ }^{12,13}$ The current recommendations of the Argentine Society of Pediatrics for use of passive prophylaxis against RSV in children include preterm infants and children with CHD. ${ }^{14}$ Although premature infant population meeting established criteria has wide access to palivizumab, universal coverage for children with $\mathrm{CHD}$ is still a challenge. Knowing the epidemiology of RSV virus infection in CHD children population in our setting will help establish more precise criteria for rational use of this high-cost drug.

The primary objective of our study was to determine hospitalization rates for ARI due to RSV and other etiologic agents and burden of disease in patients under 2 years old with hemodynamically significant CHD admitted in a multidisciplinary program for long-term outpatient followup. Secondarily we explored biological or social risk factors associated with hospitalization for ARI.

\section{Methods \\ Design}

Prospective, longitudinal, observational study in a cohort of patients with CHD followed up in an outpatient multidisciplinary program.

\section{Setting}

Hospital de Pediatría Prof. Dr. J. P. Garrahan, located in Buenos Aires, Argentina, is a tertiary referral center for high complexity patients. Only outborn patients are assisted at the neonatal intensive care unit (NICU). Eighty-five per cent of NICU patients come from low socioeconomic status families and do not have health insurance. Discharged patients are admitted in a multidisciplinary program for long-term outpatient follow-up at the high-risk clinic.

\section{Population}

Patients less than 2 years old with hemodynamically significant $\mathrm{CHD}$ on follow-up at the high-risk clinic were consecutively included between 2006 and 2008. All patients were assessed by a pediatric cardiologist. Hemodynamically significant CHD was diagnosed according to the American Academy of Pediatrics 2003 criteria, ${ }^{15}$ i.e., if one of the following was present: (1) ongoing medication for congestive heart failure, (2) moderate to severe pulmonary hypertension diagnosed by Doppler ultrasound, (3) cyanotic heart disease (oxygen saturation under 90\%). Exclusion criteria were: prior RSV passive prophylaxis with palivizumab and/or poor CHD prognosis (life expectancy less than 6 months). Elimination criterion was: patient loss during follow-up. Study completion was defined as: death, resolution of hemodynamic compromise, or reaching second birthday.

Study primary outcome measure was hospitalization for ARI caused by RSV and other agents. Secondary outcome was burden of disease in hospitalized patients, measured as length of stay, admission to intensive care unit (ICU), use of mechanical ventilation, and clinical course.

At study beginning, families were trained in preventing and detecting early signs of ARI in their children. During follow-up, reinforcement was done by one of the main researchers on regular monthly visits and through phone calls every 2 weeks. Parents were instructed to consult if a respiratory infection was suspected. ARI diagnosis and management, as well as need of hospitalization, were determined according to institutional clinical guidelines. ${ }^{16}$

Hospitalized patients were followed by one of the investigators until discharge. In-hospital days, need for intensive care, use of mechanical ventilation, and clinical course were recorded for each patient.

Viral diagnostic tests were routinely performed in every hospitalized patient. Nasopharyngeal secretions were aspirated into a mucus sample trap with an 8 or 10 Fr suction catheter attached to wall suction. Samples were immediately transported to laboratory and analyzed by rapid immunofluorescence with a polyclonal mixture. If the result was positive, the sample was matched with monoclonal antibodies to detect RSV, adenovirus, influenza A and B, and parainfluenza 1, 2 and 3 (Chemicon International, Temecula, CA). This method has a specificity of $99 \%$ and a sensibility of $72.8 \%$ (manufacturer data).

Data were collected prospectively and the following variables were considered: birth weight, gestational age, gender, type and treatment of heart disease, presence of pulmonary hypertension, siblings or children under 10 in the household, maternal schooling level, and place of 
living. Socioeconomic condition was defined according to Argentina's National Institute of Statistics and Census (Instituto Nacional de Estadística y Censos, INDEC). "Unsatisfied basic needs" refers to the following features: serious crowded living condition, inadequate or precarious housing, child at school age not attending school and/or illiterate family head. ${ }^{17}$

\section{Statistical analysis}

Data were summarized using frequency tables, averages, and measures of dispersion; incidence rates of hospitalization were calculated for overall ARI and for RSV-ARI. Because children had different follow-up periods, incidence density was additionally calculated. All measures are reported with their respective $95 \%$ confidence intervals ( $95 \% \mathrm{CI}$ ). For risk factor estimates, independent variables were compared by using $t$ test or Wilcoxon test for continuous variables, according to distribution, and by Fisher's exact test for categorical variables. We used a logistic regression model to evaluate independent risk factors. Differences with $p<0.05$ were considered as significant. The STATA 9.0 package for Windows was used for data analysis.

In all cases, either parent or patient's legal guardian confirmed his/her desire to participate in the study by signing a consent form, after being fully informed of all the aspects of the research. The study was approved by the hospital's Institutional Review Board and Ethics Committee.

\section{Results}

Of the 123 patients under 2 years old with CHD followed at the High Risk Clinic during the study period, 71 met eligibility criteria. All of them were enrolled and form the study cohort. No patient met exclusion criteria. This cohort was followed up from 1 to 22 months, with a median of 6 months; one patient was lost to follow-up. Six children died during follow-up as a result of their heart disease. Cohort characteristics are summarized in Table 1.

Seventy-four percent of children (52/71) were treated surgically, but in most patients (75\%) surgery was palliative; 33 children (47\%) required drug therapy for their heart disease and 25 patients (36\%) had pulmonary hyperflow.

Children entered the study at different ages; 60 children (85\%) were less than 6 months old at start of follow-up. In the country, RSV epidemic season is between May and September; 39 children (54\%) entered the study during these high-risk months.

Twenty-two children were hospitalized for ARI, 15 (68\%) due to RSV. Overall ARI hospitalization rate was 22/71 or $31 \%$ (95\%CI $20-43 \%$ ), while RSV-ARI hospitalization rate was $15 / 71$ or $21 \%$ ( $95 \%$ CI $12-32 \%)$. The cohort was followed up for 543 months (16,290 days); incidence density for overall ARI hospitalization was 1.35 episodes per 1,000 days of follow-up, while RSV-ARI incidence density was 0.92 episodes per 1,000 days of follow-up (RSV-ARI incidence density adds up to 3.42 episodes per 1,000 days considering just RSV season months).

Table 1 - Cohort characteristics $(n=71)$

\begin{tabular}{lc}
\hline Variável & $\mathbf{n ~ ( \% )}$ \\
\hline Birth weight $(\mathrm{g})$ & $3,125(980-4,730)^{*}$ \\
GA (weeks) & $39(31-42)^{*}$ \\
Prematurity (<37 weeks GA) & $8(11 \%)$ \\
Male sex & $45(63 \%)$ \\
Age at recruitment (months) & $3(1-19)^{*}$ \\
Most common diagnosis & $17(24 \%)$ \\
$\quad$ Left or right ventricle hypoplasia & $16(22.5 \%)$ \\
$\quad$ Atrial septal defect & $1(1.5 \%)$ \\
Surgery $\quad$ Palliative & $52(74 \%)$ \\
$\quad$ Corrective & $39(75 \%)$ \\
Pulmonary hypertension & $13(25 \%)$ \\
Breastfeeding & $13(19 \%)$ \\
Home smoking & $37(52 \%)$ \\
Marental asthma & $35(49 \%)$ \\
Siblings < 10 years & $11(15 \%)$ \\
Unsatisfied basic needs & $27(39 \%)$
\end{tabular}

$\mathrm{GA}=$ gestational age.

* Median (range). 
Seasonal distribution of severe cases with hospitalization is depicted in Figure 1.

Hospitalization for ARI due to RSV occurred from May through September (mid-fall to mid-winter), with a maximum peak in June and sporadic cases in other months. No consistent pattern distribution was observed for other viruses. Main characteristics and outcomes during hospitalization for RSV-ARI and non-RSV-ARI groups are shown in Table 2. Children with RSV presented a more complicated clinical course with more prolonged hospital stays, one third of them needing admission to ICU and mechanical ventilation, although these differences did not reach statistical significance. No child died from ARI or had sequelae at discharge. Respiratory complications in this group caused neither hemodynamic impairment nor cancellation of scheduled surgical intervention.

Table 3 depicts baseline characteristics and risk factors among controls (patients in cohort without hospitalized ARI) and hospitalized groups (RSV and non-RSV). Comparisons by bivariate analysis show that patients in RSV group were younger and more frequently breastfed than controls. No other variable reached statistical significance. In multivariate analysis, younger age was an independent risk factor for severe RSV-ARI, while breastfeeding effect disappears, which most probably reflects a confounding effect related to young age.

\section{Discussion}

Lower ARI has been associated with significant morbidity and mortality in children with $\mathrm{CHD}$ and hemodynamic compromise ${ }^{5-7,18}$. Infection with RSV appears to be significantly more severe compared to other respiratory viruses $8,9,19-22$. Our study results are consistent with this evidence. While in healthy population less than $3 \%$ of infants without risk factors require hospitalization for ARI, in our study $31 \%$ of children did, in most cases due to RSV. In a recent publication, Meissner et al. estimated that the risk of hospitalization for RSV infection in patients aged under 2 years with CHD and hemodynamic compromise who did not receive palivizumab prophylaxis was five to seven times higher than in the general pediatric population 22 ; our study included children with similar characteristics, and the risk found is in agreement with the upper range described by the authors. Similar findings have been reported in our region regarding another high-risk pediatric population, namely that of former premature infants with and without bronchopulmonary dysplasia. 23-26

Estimated rates of hospitalization and mortality due to ARI in children with heart disease came from studies considering overall number of pediatric patients hospitalized for ARI as denominator; our study instead estimated incidence in a cohort of patients with CHD. Two large multicenter trials in children aged under 2 years with $\mathrm{CHD}$ serve as reference for rate comparison. In the randomized, controlled, double-blind study conducted by Feltes et al. ${ }^{27}$ to evaluate the efficacy of palivizumab, 1,287 patients from 76 centers with diagnosis of CHD and hemodynamic compromise were included; RSV hospitalization rate among those who received palivizumab $(n=639)$ was $5.3 \%$ vs. $9.7 \%$ in the placebo group ( $n=648$ ). In the descriptive study conducted by Medrano López et al. in 53 hospitals in Spain, 1,248 children with similar criteria were studied; only $5.8 \%(n=72)$ did not receive RSV prophylaxis; in this group, RSV-ARI hospitalization rate was $6.8 \% .{ }^{28}$

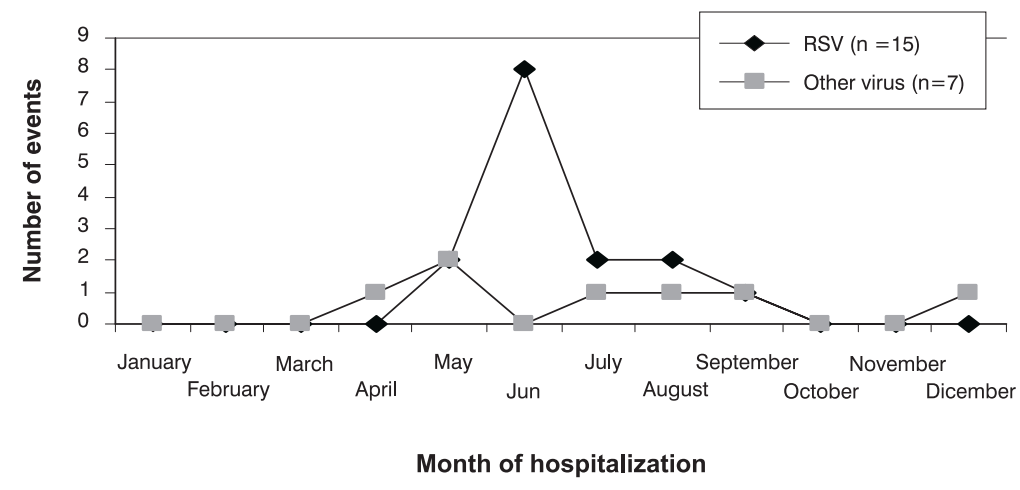

$\mathrm{RSV}=$ respiratory syncytial virus.

Figure 1 - Seasonal distribution, hospitalizations for acute respiratory infection 
In our study, hospitalization rate and incidence density were higher than those reported by Feltes and Medrano López, even considering that these authors' findings correspond to follow-up during RSV season and, in our study, follow-up was continued while hemodynamic compromise was present or until second birthday was reached. We speculated that a recruitment bias could explain our results but were not able to find one. The proportion of patients with the three more common cardiologic diagnosis was similar in our study and in Feltes et al. ${ }^{27}$
Regarding disease burden, mean hospital stay was 22 days (range 1-88) among RSV hospitalized children, $40 \%$ of them were admitted to the pediatric ICU and $30 \%$ needed mechanical ventilation. In a prior study conducted at the same hospital, Giubergia et al.29 found that $6.3 \%$ of infants without risk factors hospitalized for RSV-ARI needed mechanical ventilation. Children with CHD hospitalized for ARI, particularly due to RSV, suffer higher morbidity than the general pediatric population. Our findings are in agreement with those of other

Table 2 - Hospitalized patients with ARI, clinical severity

\begin{tabular}{|c|c|c|c|}
\hline Variable & $\begin{array}{l}\text { RSV-ARI } \\
(n=15)\end{array}$ & $\begin{array}{c}\text { Non-RSV-ARI } \\
(n=7)\end{array}$ & $\mathbf{p}$ \\
\hline Age (months)* & $7(1-21)$ & $6(4-13)$ & 0.16 \\
\hline Days hospitalized* & $22(1-88)$ & $6(2-11)$ & 0.14 \\
\hline ICU $_{\text {admission }}{ }^{\dagger}$ & $6(40 \%)$ & $1(14 \%)$ & 0.35 \\
\hline Mechanical ventilation ${ }^{\dagger}$ & $5(30 \%)$ & 0 & 0.13 \\
\hline Sequelae $^{\dagger}$ & 0 & 0 & - \\
\hline Death $^{+}$ & 0 & 0 & - \\
\hline
\end{tabular}

$\mathrm{ARI}=$ acute respiratory infection; ICU = intensive care unit; RSV = respiratory syncytial virus.

* Median (range).

$\dagger \mathrm{n}(\%)$.

Table 3 - Comparative characteristics of hospitalized patients (RSV and non-RSV) and non-hospitalized controls

\begin{tabular}{|c|c|c|c|c|}
\hline Variables & $\begin{array}{l}\text { Controls } \\
(n=49) \\
n(\%)\end{array}$ & $\begin{array}{c}\text { RSV-ARI } \\
(n=15) \\
n(\%)\end{array}$ & $\begin{array}{c}\text { Non-RSV-ARI } \\
(n=7) \\
n(\%)\end{array}$ & $\mathbf{p}$ \\
\hline Age (months) & $4.5 \pm 4.1 *$ & $1.9 \pm 1.2^{*}$ & $4.5 \pm 2.3^{*}$ & 0.04 \\
\hline Male sex & $30(61 \%)$ & $11(73 \%)$ & $4(57 \%)$ & NS \\
\hline Birth weight $(\mathrm{g})$ & $3,013 \pm 709 *$ & $3,100 \pm 712 *$ & $3,170 \pm 795^{*}$ & NS \\
\hline Preterm birth & $5(10 \%)$ & $3(20 \%)$ & - & NS \\
\hline NICU stay (days) & $52 \pm 37 *$ & $45 \pm 22 *$ & $65 \pm 14^{*}$ & NS \\
\hline Maternal age (years) & $27 \pm 7 *$ & $24 \pm 6 *$ & $27 \pm 6 *$ & NS \\
\hline Breastfeeding & $22(45 \%)$ & $12(80 \%)$ & $3(43 \%)$ & 0.05 \\
\hline Maternal schooling $\leq 7$ years & $17(35 \%)$ & $7(47 \%)$ & $3(43 \%)$ & NS \\
\hline Unsatisfied basic needs & $3(6 \%)$ & $3(20 \%)$ & $2(28 \%)$ & NS \\
\hline Home smoking & $24(49 \%)$ & $9(60 \%)$ & $2(29 \%)$ & NS \\
\hline Parental asthma & $10(20 \%)$ & - & $1(14 \%)$ & NS \\
\hline Siblings $<10$ years & $32(67 \%)$ & $11(73 \%)$ & $6(86 \%)$ & NS \\
\hline Previous cardiac surgery & $34(71 \%)$ & $11(73 \%)$ & $7(100 \%)$ & NS \\
\hline Pulmonary hypertension & $10(21 \%)$ & $3(20 \%)$ & - & NS \\
\hline
\end{tabular}

$\mathrm{ARI}=$ acute respiratory infection; NICU = neonatal intensive care unit; NS = not significant; RSV = respiratory syncytial virus.

* Mean \pm standard deviation. 
authors, $7,9,18$ with figures even higher than those by Navas et al., ${ }^{18}$ who reported $33.4 \%$ and $18.8 \%$ for admission to ICU and need for mechanical ventilation, respectively. Disease burden was higher for the RSV group compared to non-RSV patients. Although these differences did not reach statistical significance, they are clinically relevant, and we speculate that the small sample size precluded reaching statistical significance.

We found no differences between type of heart disease and risk of hospitalization. Other authors have reported an increased risk in the group of patients with pulmonary hypertension. ${ }^{30}$ Genetic factors, number of surgeries, and presence of a shunt have also been described as risk factors of hospitalization for ARI in other studies, 20 but were not explored in ours.

Mortality in our series was higher than in others, though no death was attributable to ARI. The mortality reported by Feltes et al. ${ }^{27}$ for placebo group was $4.2 \%$ (half the figure in our study) and mortality attributable to ARI was $0.3 \%$.

Finally, when risk factors for RSV hospitalization in our population were explored by multivariate analysis, the only factor found was younger age in months; this risk factor has also been found in general population studies conducted in our setting. ${ }^{29}$ There are many reasons why younger children are more prone to develop severe ARI, including certain anatomical and physiological characteristics of the respiratory tract, particularly smaller airway size and lower lung compliance.

In conclusion, our data show that, in our country, the impact of RSV children with CHD, measured as need for hospitalization, is doubling that reported in patients without immunoprophylaxis in industrialized countries. Disease burden associated with RSV-ARI was considerable, although no patient developed sequelae or died from ARI in the study group. Except younger age, no other biological or social risk factors were found associated with RSV-ARI hospitalization in this cohort of children with CHD.

\section{References}

1. Avila MM, Carballal G, Salomón H, Cerqueiro MC, Catalano M, Messad OS, et al. Lower respiratory tract infections in children under five years in Buenos Aires. Infect Microbiol Clin. 1990;2:38-47.

2. Rodrigues $F E$, Tatto RB, Vauchinski L, Leães $L M$, Rodrigues $M M$, Rodrigues VB, et al. Pneumonia mortality in Brazilian children aged 4 years and younger. J Pediatr (Rio J). 2011;87:111-4.

3. Salomão Junior JB, Gardinassi LG, Simas PV, Bittar CO, Souza FP, Rahal $P$, et al. Human respiratory syncytial virus in children hospitalized for acute lower respiratory infection. J Pediatr (Rio J). 2011;87:219-24.

4. Dirección Nacional de Maternidad e Infancia. Abril 2009. http:// www.msal.gov.ar/promin/. Access: 01/01/2012.

5. García CG, Bhore R, Soriano-Fallas A, Trost M, Chason R, Ramilo $\mathrm{O}$, et al. Risk factors in children hospitalized with RSV bronchiolitis versus non-RSV bronchiolitis. Pediatrics. 2010;126:e1453-60.
6. Wang EE, Law BJ, Stephens D. Pediatric Investigators Collaborative Network on Infections in Canada (PICNIC) prospective study of risk factors and outcomes in patients hospitalized with respiratory syncytial viral lower respiratory tract infection. J Pediatr. 1995; 126:212-9.

7. Khongphatthanayothin A, Wong PC, Samara Y, Newth CJ, Wells WJ, Starnes VA, et al. Impact of respiratory syncytial virus infection on surgery for congenital heart disease: postoperative course and outcome. Crit Care Med. 1999;27:1974-81.

8. Moler FW, Khan AS, Meliones JN, Custer JR, Palmisano J, Shope TC. Respiratory syncytial virus morbidity and mortality estimates in congenital heart disease patients: a recent experience. Crit Care Med. 1992;20:1406-13.

9. MacDonald NE, Hall CB, Suffin SC, Alexson C, Harris PJ, Manning JA. Respiratory syncytial viral infection in infants with congenital heart disease. N Engl J Med. 1982;307:397-400.

10. Magliola R, Laura J, Capelli H. Current state of children with congenital heart defects in Argentina. Arch Argent Pediatr. 2000;98:130-3.

11. Magliola R, Althabe M, Charroqui A, Moreno G, Balestrini M, Landry L, et al. Congenital heart disease: surgical results in a pediatric public hospital 1994-221. Arch Argent Pediatr. 2004;102:110-4.

12. Committee on Infectious Diseases. From the American Academy of Pediatrics: Policy statements - Modified recommendations for use of palivizumab for prevention of respiratory syncytial virus infections. Pediatrics. 2009;124:1694-701.

13. Medrano López C, García-Guereta L, Fernández Pineda L, Malo Concepción P, Maroto Alvaro E, Santos de Soto J, et al. Clinical consensus on respiratory syncytial virus (RSV) infection prophylaxis and the use of palivizumab in paediatric cardiology. An Pediatr (Barc). 2010;72:432.e1-13.

14. Comité de Estudios Fetoneonatales (CEFEN). Update on the recommendations for the use of palimizumab. Arch Argent Pediatr. 2007; 105:67-70.

15. American Academy of Pediatrics Committee on Infectious Diseases and Committee on Fetus and Newborn. Revised indications for the use of palivizumab and respiratory syncytial virus immune globulin intravenous for the prevention of respiratory syncytial virus infections. Pediatrics. 2003;112:1442-6.

16. Atención de Pacientes con Enfermedades Respiratorias. Criterios de Atención. Hospital de Pediatría Prof. Dr. Juan P Garrahan. 2000:62-8. http://172.16.200.7/cpw/intracrite/crivol2.pdf. Access: 02/01/2012.

17. Instituto Nacional de Estadística y Censos (INDEC). La pobreza en la Argentina: asociación de características de los hogares con mediciones de la pobreza y diseño de un indicador compuesto de necesidades básicas insatisfechas. Buenos Aires: INDEC; 1985; 1 :Ed 2.

18. Navas $L$, Wang $E$, de Carvalho V, Robinson J. Improved outcome of respiratory syncytial virus infection in a high-risk hospitalized population of Canadian children. Pediatric Investigators Collaborative Network on Infections in Canada. J Pediatr. 1992; 121:348-54.

19. Hon KL, Leung TF, Cheng WY, Ko NM, Tang WK, Wong WW, et al. Respiratory syncytial virus morbidity, premorbid factors, seasonality, and implications for prophylaxis. J Crit Care. 2012 Jan 6.

20. Medrano C, Garcia-Guereta L, Grueso J, Insa B, Ballesteros F, Casaldaliga J, et al. Respiratory infection in congenital cardiac disease. Hospitalizations in young children in Spain during 2004 and 2005: the CIVIC Epidemiologic Study. Cardiol Young. 2007; 17:360-71.

21. Sociedad Argentina de Pediatría. Recommendation for the diagnosis and treatment of acute lower respiratory infections in children under two years. Arch Argent Pediatr. 2006;104:159-76.

22. Meissner HC, Bocchini JA Jr, Brady MT, Hall CB, Kimberlin DW, Pickering LK. The role of immunoprophylaxis in the reduction of disease attributable to respiratory syncytial virus. Pediatrics. 2009;124:1676-9. 
23. Bauer G, Dussel V, Fariña D, Rodríguez S. Respiratory syncitial virus infection in vulnerable population: biological risk versus social risk. Arch Argent Pediatr. 2005;103:198-204.

24. Klein MI, Coviello S, Bauer G, Benitez A, Serra ME, Schiatti MP, et al. The impact of infection with human metapneumovirus and other respiratory viruses in young infants and children at high risk for severe pulmonary disease. J Infect Dis. 2006;193:1544-51.

25. Fariña D, Rodríguez SP, Bauer G, Novali L, Bouzas L, González $\mathrm{H}$, et al. Respiratory syncytial virus prophylaxis: cost-effective analysis in Argentina. Pediatr Infect Dis J. 2002;21:287-91.

26. Rodríguez SP, Fariña D, Bauer G. Respiratory syncytial virus prophylaxis in a high-risk population in Argentina: a costeffectiveness analysis. Pediatr Infect Dis J. 2008;27:660-1.

27. Feltes TF, Cabalka AK, Meissner HC, Piazza FM, Carlin DA, Top $\mathrm{FH} \mathrm{Jr}$, et al. Palivizumab prophylaxis reduces hospitalization due to respiratory syncytial virus in young children with hemodynamically significant congenital heart disease. J Pediatr. 2003;143:532-40.
28. Medrano López C, García-Guereta Silva L. Respiratory infections and congenital heart disease: two seasons of the CIVIC study. An Pediatr (Barc). 2007;67:329-36.

29. Giubergia V, Martinchuk G, Moreno N, Colombres G, Parra L, Viale $D$, et al. Severity of synctitial respiratory virus infection in patients with risk factors and without them. Arch Argent Pediatr. 2004; 102:330-5

30. Meissner HC. Selected populations at increased risk from respiratory syncytial virus infection. Pediatr Infect Dis J. 2003;22:S40-4.

Correspondence:

Silvia Andres, MD

Calle 37, no. 372, Dto 4, La Plata

1900 - Buenos Aires - Argentina

Tel. : +54 (221) 427.5747, +54 (221) 555.7211

E-mail: silviabandres@hotmail.com 